\title{
Lipoma intracraniano - Revisão da literatura
}

\author{
Carlos Umberto Pereira', Antonio Carlos Azevedo Silveira², Alberto Silva Barreto ${ }^{3}$, \\ Allan Valadão de Oliveira Britto ${ }^{4}$, Breno José Alencar Pires Barbosa ${ }^{5}$ \\ Serviço de Neurocirurgia do Hospital de Urgência de Sergipe, Aracaju, SE, Brasil. \\ Hospital São Lucas e Hospital Primavera, Aracaju, SE, Brasil.
}

\section{RESUMO}

Lipomas intracranianos são considerados malformações congênitas, resultantes da persistência da meninge primitiva mesenquimal e de sua posterior diferenciação em tecido adiposo. Correspondem entre $0,06 \%$ e $0,1 \%$ de todos os tumores intracranianos. Exame de neuroimagem tem sido útil no seu diagnóstico em pacientes assintomáticos. Geralmente, encontra-se associado a outras anomalias congênitas como agenesia do corpo caloso ou representa achado ocasional relacionado a outras manifestações clínicas não correlatas. Tratamento cirúrgico não é recomendado por causa da elevada taxa de complicações e pelo curso benigno dessa lesão. Este trabalho teve como delineamento uma revisão bibliográfica nas bases de dados online Cochrane, PubMed/MedLine, Lilacs e SciELO, para artigos publicados entre os anos de 1974 e 2010. Os descritores utilizados foram: "lipoma cerebral" e "lipoma intracraniano", combinados com "tumor cerebral" e "tratamento". Foram identificados 94 artigos, dos quais, após a leitura na íntegra e análise pelos autores, foram selecionados 75 artigos para este estudo. Os lipomas intracranianos resultam da persistência e diferenciação anômala da meninge primitiva em tecido adiposo. No exame tomográfico, apresenta-se como lesão marcadamente hipoatenuante, sem realce significativo pelo contraste endovenoso, não apresentando edema perilesional. A ressonância magnética tem sido o exame de eleição para o seu diagnóstico. Quando decorrente de achado incidental e assintomático, seu tratamento é conservador. Lipoma intracraniano é uma malformação rara resultante de alterações do desenvolvimento e encontra-se frequentemente associado a disrafismos. Geralmente é assintomático ou um achado incidental de exame de imagem. Os avanços nos métodos de diagnóstico por imagem aumentaram a probabilidade de essa malformação ser diagnosticada, mesmo que assintomática. Seu tratamento é conservador em casos assintomáticos ou de achado incidental.

\section{PALAVRAS-CHAVE}

Neoplasias encefálicas, lipoma/congênito, lipoma/diagnóstico, terapêutica, tumor cerebral, lipoma intracraniano, tratamento.

\section{ABSTRACT}

Intracranial lipoma - Review of literature

Intracranial lipomas are considered to be congenital malformations, originated from primitive mesenchymal meninge persistence and later differentiation into fatty tissue. They represent $0.06 \%$ to $0.1 \%$ of all intracranial tumors. Neuroimaging is useful for diagnosing symptomatic patients. The lesion is often associated with other congenital anomalies such as agenesis of the corpus callosum, however it may be found incidentally with atypical clinical manifestations. Surgical treatment is not recommended due to high complication rates and to the condition's benign course. The present paper reviews the literature through the online databases Cochrane, PubMed/MedLine, Lilacs and SciELO. The reviewed articles were published from 1974 to 2010; descriptors included "cerebral lipoma" and "intracranial lipoma" associated to "brain tumor" and/or "treatment". Intracranial lipomas result from primitive mesenchymal meninge's persistence and later abnormal differentiation into fatty tissue. Computed tomography scan reveals a hypodense lesion, with no enhancement after intravenous application of contrast media or perilesional edema. Magnetic resonance imaging is currently the best method for diagnosis. In case of incidental finding in a previous asymptomatic patient, no surgical treatment is indicated. Intracranial lipomas are rare malformations originated from development abnormalities and usually associated with dysraphisms. This lesion is often either asymptomatic or an incidental imaging finding. Improvements in the neuroimaging field may lead to higher diagnostic rates, even in asymptomatic individuals. No surgical treatment is indicated for asymptomatic or incidental cases.

\section{KEYWORDS}

Brain neoplasms, lipoma/congenital, lipoma/diagnosis, therapeutics, brain tumors, intracranial lipomas, treatment.

1 Professor doutor do Departamento de Medicina da Universidade Federal de Sergipe (UFS), Serviço de Neurocirurgia do Hospital de Urgência de Sergipe, Aracaju, SE, Brasil.

2 Neurocirurgião, Serviço de Neurocirurgia do Hospital de Urgência de Sergipe, Aracaju, SE, Brasil.

3 Neurocirurgião do Hospital São Lucas e Hospital Primavera, Aracaju, SE, Brasil.

4 Médico radiologista do Hospital de Urgência de Sergipe, Aracaju, SE, Brasil.

5 Médico-residente de Cirurgia Geral do Hospital da Restauração, Recife, PE, Brasil. 


\section{Introdução}

Lipomas intracranianos (LIC) são tumores adiposos, benignos e raros..$^{1-7}$ Raramente provocam sintomas e frequentemente são um achado incidental. ${ }^{4,8-14}$ Geralmente estão associados a outras anomalias congênitas ou representam achados ocasionais relacionados a outras manifestações clínicas não correlatas. ${ }^{10,11,15-17}$ Os avanços nas técnicas de imagem têm aumentado a probabilidade de sua identificação ao longo da vida. ${ }^{6,10,18-20}$

\section{Epidemiologia}

Foi descrito pela primeira vez por Rokitansky, ${ }^{21} \mathrm{em}$ 1856, um lipoma localizado no corpo caloso e associado à agenesia do corpo caloso, em um achado de autópsia. Corresponde entre $0,06 \%$ e $0,1 \%$ de todos os tumores intracranianos. ${ }^{2-4,22,23} \mathrm{O}$ LIC tem sido considerado como uma lesão comum no adulto. Recentemente, com o uso de métodos modernos de imagens como ultrassom (US) e ressonância magnética (RM), são diagnosticados cada vez mais na infância., ${ }^{47-19,24-26}$

\section{Localização}

As localizações mais frequentes são: inter-hemisférica (56\%) - geralmente pericaloso -, cisterna quadrigeminal (25\%), suprasselar/interpeduncular (14\%), ângulo pontocerebelar (9\%) e cisterna silviana (5\%). ${ }^{2,4,6,9,11,15,23,27-31}$ Outras localizações descritas foram: região pineal, ${ }^{32}$ lobo frontal, ${ }^{33}$ ângulo pontocerebelar, ${ }^{34-39}$ plexo coroide, ${ }^{40-43}$ cerebelo, ${ }^{44}$ bulbo, ${ }^{45,46}$ septum pellucium $^{13}$ e intraventricular. ${ }^{26,41} \mathrm{Tem}$ sido descrita a presença concomitante de lipoma extra e intracraniano. ${ }^{19,47-50}$

Lipoma da região da cisterna quadrigêmina tem sido relatado como lipoma da cisterna quadrigeminal, placa quadrigeminal, cisterna ambiens, vermis superior ou do velum medular superior. ${ }^{51}$

\section{Etiopatogenia}

Os LIC são atualmente considerados malformações congênitas, resultantes da persistência da meninge primitiva mesenquimal e de sua posterior diferenciação em tecido adiposo. ${ }^{5,11,13,20,25,52-54}$ Vários autores acreditam que são tumores de inclusão por se localizarem na linha média e frequentemente se relacionarem com distúrbios do fechamento do tubo neural ${ }^{48,53}$ Para outros autores, seriam uma falha na diferenciação do tecido meníngeo primitivo na fissura inter-hemisférica, disgenesia do mesênquima meníngeo ou vascular in situ, que secundariamente interfeririam com o desenvolvimento das estruturas da linha média. ${ }^{31,52}$ Poucos casos foram relatados entre lipomas do couro cabeludo e intracraniano, o que pode demandar investigação por imagem em crianças pequenas portadoras do primeiro achado ${ }^{47,50,55}$ Múltiplos LIC associados a lesões cutâneas faciais e orbitárias demandam avaliação imediata para uma síndrome subjacente, como a lipomatose encefalocraniocutânea..$^{50,56,57}$

Os LIC podem estar associados a várias malformações cerebrais, como displasia ou agenesia do corpo caloso, encefalocele, displasia frontonasal ou cheiliquisis, cranium bifidum, agenesia do vermis cerebelar, microgiria, ectopia cranial ou espinha bífida. .10,22,58 $^{2}$

\section{Classificação anatômica do lipoma do corpo caloso}

Truwit e Barkovich, ${ }^{25}$ estudando os achados de ressonância magnética (RM) em 42 pacientes com LIC, classificaram o lipoma do corpo caloso em dois tipos: tubulonodular e o curvilíneo. O curvilíneo pode ser pequeno ou extenso, localizado predominantemente posterior e assintomático. ${ }^{6} \mathrm{O}$ tubulonodular pode ser predominantemente localizado anteriormente, volumoso e frequentemente associado com malformações. ${ }^{6}$ O subtipo anterior parece ser a forma mais severa de lipoma tubulonodular.

\section{Histopatologia}

Do ponto de vista macroscópico, apresenta consistência mole, móvel e indolor. Consiste em tecido adiposo maduro, com quantidade variada de colágeno em pontos de contato com tecido nervoso e variados graus de vascularização. ${ }^{22} \mathrm{O}$ padrão de crescimento dos lipomas geralmente é mais próximo ao dos hamartomas que ao das demais neoplasias. Podem fazer parte de teratomas, apresentar componente osteocartilaginoso, ${ }^{53,59}$ ou ninhos de proliferação de células de Schwann. Calcificação pode ocorrer e, nesse caso, são denominados de osteolipomas. Estes se desenvolvem geralmente na região suprasselar/interpeduncular, sendo caracterizados por um arranjo adiposo central e tecido ósseo periférico. ${ }^{33}$ 


\section{Quadro clínico}

Geralmente têm desenvolvimento lento e são assintomáticos. ${ }^{12,60}$ Dependendo da localização e do tamanho, podem se manifestar com cefaleia, crise convulsiva, paralisia ou retardo mental, especialmente quando presentes no corpo caloso. ${ }^{16,61}$ As manifestações clínicas estão relacionadas com a compressão das estruturas adjacentes relacionadas com a lesão, comprometimento de nervos cranianos, vasos e sistema ventricular, com consequente déficit neurológico focal, hidrocefalia obstrutiva e elevação da pressão intracraniana. Quando localizados na cisterna quadrigeminal ou na cisterna ambiens, produzem sintomatologia em $20 \%$ dos casos com o comprometimento da circulação liquórica e causam sinais de hipertensão intracraniana, hidrocefalia, distúrbios da motilidade ocular e crises convulsivas. ${ }^{4,16,61-63}$ Quando localizados no hemisfério cerebral, produzem mais sintomas em relação aos localizados na linha média. ${ }^{8,25}$ Anormalidades endocrinológicas são raras. ${ }^{10}$ Lipomas localizados na fissura silviana podem causar epilepsia. ${ }^{3,6,10,28,30,54,64}$ Hädecke et al ${ }^{65}$ relataram um paciente de 29 anos que tinha crises parciais complexas por 19 anos, cujo exame de imagem revelou a presença de múltiplos lipomas intracranianos. Zimmermann et al. ${ }^{66}$ descreveram um caso de lipoma localizado no ângulo pontocerebelar em uma paciente de 27 anos, com história prévia de dois meses de vertigem e perda auditiva progressiva.

\section{Exames de neuroimagem}

As imagens características dos LIC pela tomografia computadorizada (TC) e ressonância magnética (RM) são consideradas patognomônicas e permitem diagnóstico preciso sem necessidade de biópsia para confirmação. ${ }^{1} \mathrm{Na}$ TC, as lesões se mostram bem circunscritas e hipoatenuantes (isoatenuantes em relação à gordura subcutânea), sem realce pelo contraste e sem edema perilesional. ${ }^{3,55,67-69} \mathrm{~A}$ mensuração por unidades Hounsfield (HU) demonstra valores típicos, variando entre -30 e $-100^{7,10,50,60}$ (Figura 1A-B). Calcificações podem ser vistas na periferia da lesão, contidas numa cápsula fibrosa que delimita o lipoma ${ }^{6,26,60}$ (Figura 2). Esse padrão é típico do lipoma do corpo caloso. ${ }^{6} \mathrm{~A}$ RM permite um diagnóstico mais preciso, especialmente em se tratando de lesões pequenas. ${ }^{12,68,70,71}$ Nas sequências em $\mathrm{T} 1$, as lesões aparecem hiperintensas, enquanto em T2 se apresentam com sinal de intensidade intermediária ou hipointensas ${ }^{2,3,7,12,25}$ (Figuras 3A-D, 4A,B). Novas sequências com saturação de gordura ou STIR (short tau inversion recovery) demonstram supressão de sinal dentro do tumor, confirmando a presença de gordura nessas lesões. ${ }^{12}$ Portanto, o diagnóstico de LIC por meio de exames de imagem é frequentemente definitivo e, na maioria dos casos, não necessita de confirmação histopatológica. ${ }^{4,30}$

A

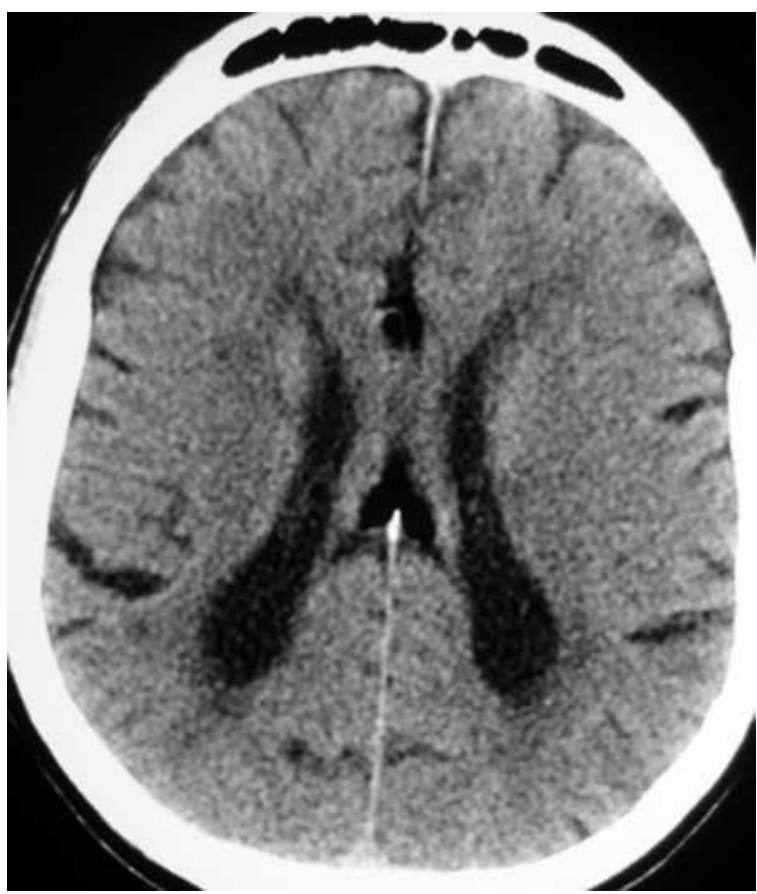

B

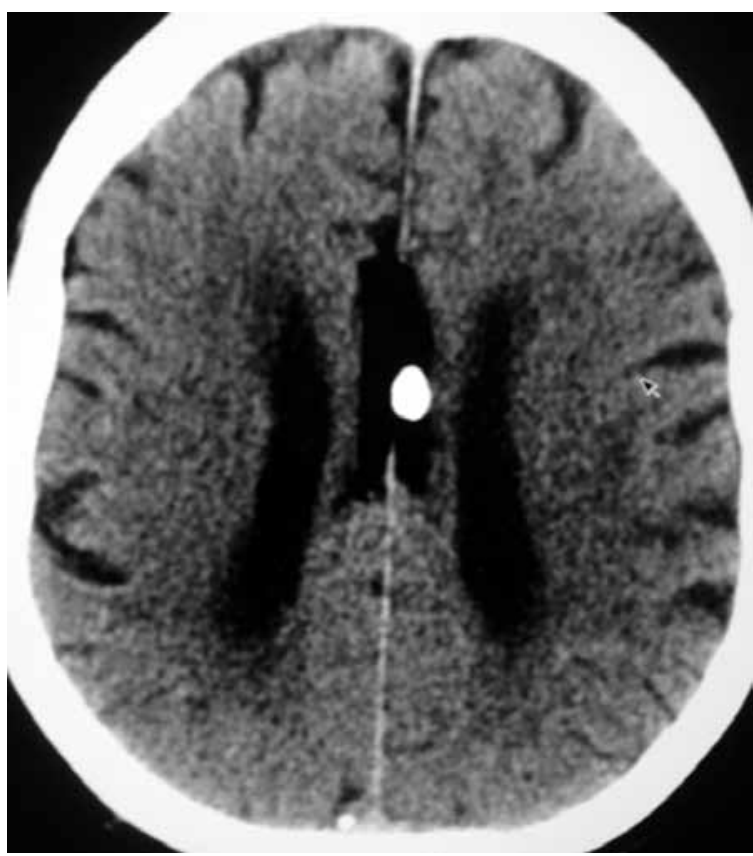

Figura 1 (A, B) - Tomografia computadorizada do crânio, plano transverso. Agenesia de corpo caloso, observando-se na sua topografia formação expansiva hipoatenuante (semelhante à da gordura subcutânea e inferior à do liquor), compatível com lipoma. Nota-se calcificação grosseira central. 


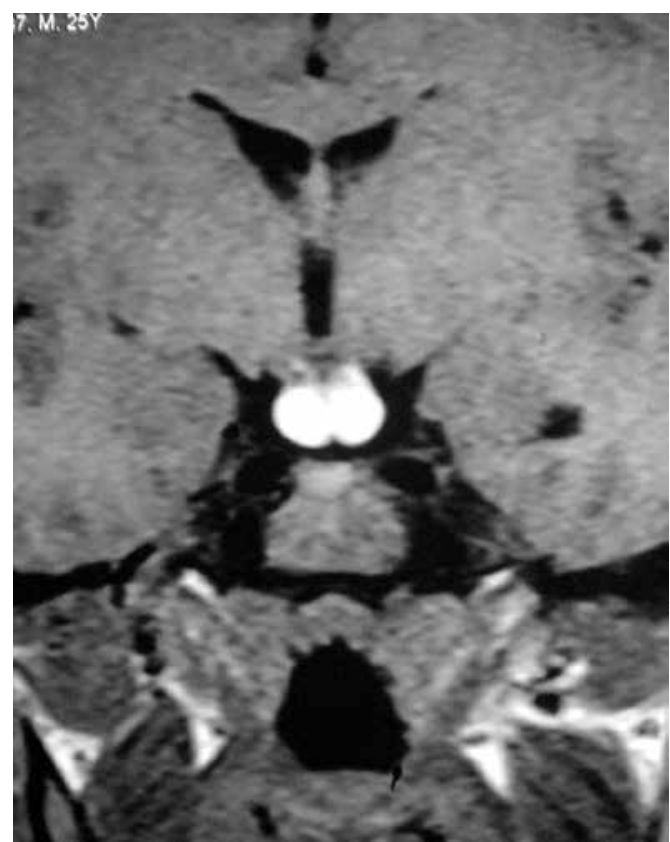

Figura 2 - Ressonância magnética do crânio, plano coronal, ponderado em T1. Imagem nodular com intenso hipersinal homogêneo, de contornos lobulados e bem definidos, localizado na cisterna suprasselar, justaquiasmática, compatível com lipoma.

A

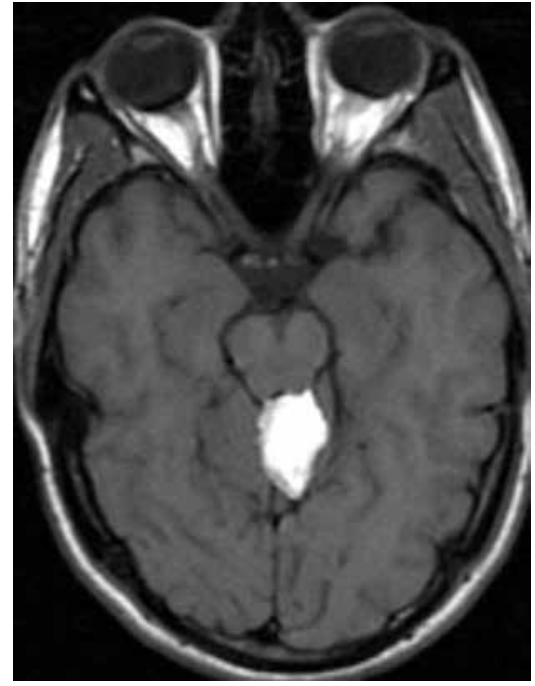

C

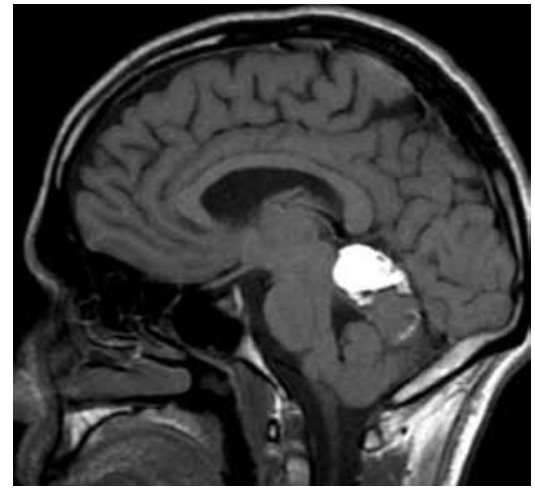

B

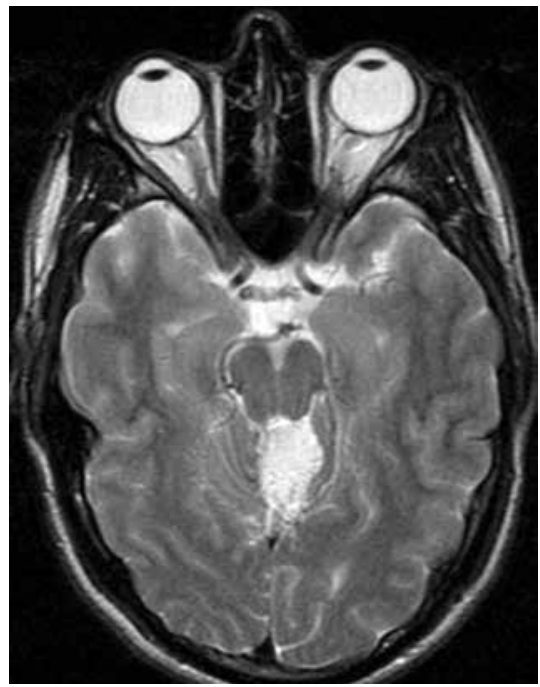

D

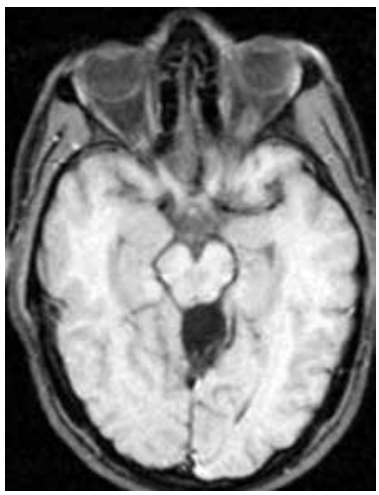

Figura 3 - Ressonância magnética do crânio (A) plano transverso ponderado em T1; (B) transverso em T2; (C) sagital em T1; (D) transverso em T1 com supressão da gordura. Nas cisternas quadrigeminal e supracerebelar, observa-se formação expansiva com intenso hipersinal

homogêneo em T1, moderado hipersinal em T2 e acentuada redução do sinal em sequência com supressão da gordura, de contornos irregulares e parcialmente definidos, com focos que se estendem até os sulcos no vermis cerebelar, compatível com lipoma. 
A

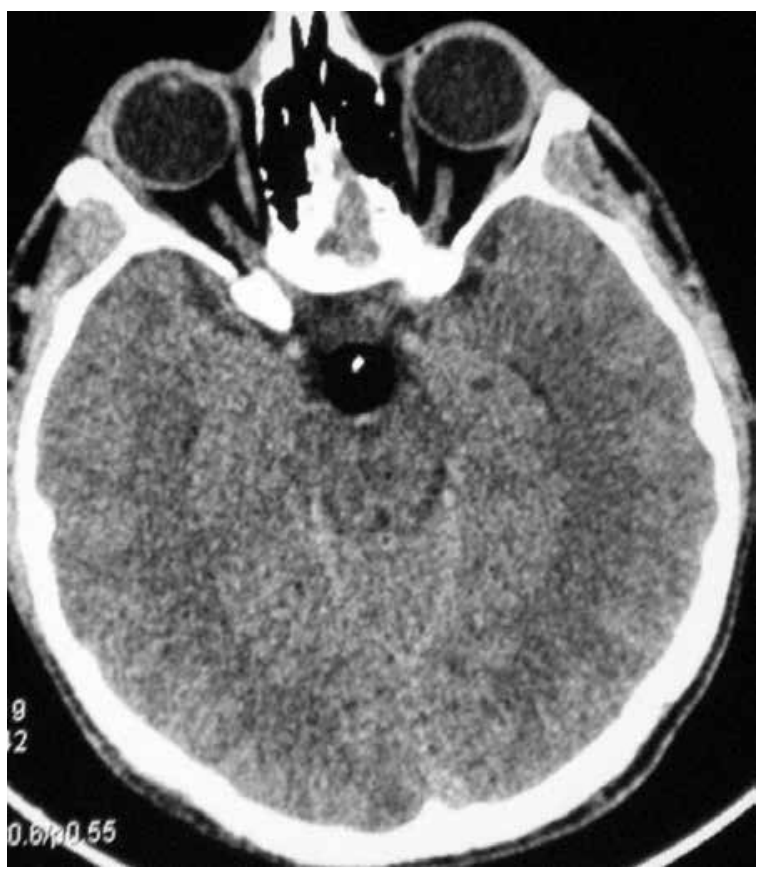

B

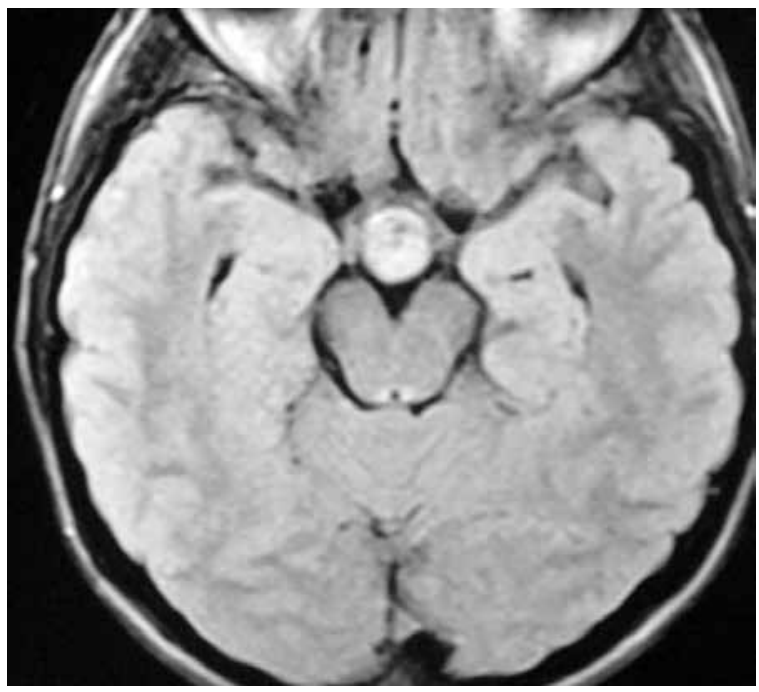

Figura 4 - (A) TC de crânio, plano transverso; (B) RM do crânio, plano transverso, ponderado em T1. Na cisterna suprasselar observa-se imagem nodular marcadamente hipoatenuante (-70 UH), com pequeno foco cálcico central, que em T1 apresenta hipersinal, compatível com lipoma.

\section{Diagnóstico diferencial}

O LIC apresenta diagnóstico diferencial com outras lesões localizadas na linha média, como cisto dermoide, teratoma do corpo caloso e outras neoplasias como germinomas. ${ }^{17}$ Diagnóstico diferencial é feito com cistos dermoides e teratomas, que podem apresentar padrões de TC similares, com acentuada hipoatenuação. ${ }^{3,6,10,50,65}$ $\mathrm{Na} \mathrm{RM}$ o cisto dermoide apresenta-se hiperintenso em $\mathrm{T} 1$ e com padrão de sinal heterogêneo em T2 com efeito expansivo; ${ }^{3,14}$ o teratoma demonstra um sinal mais heterogêneo e pode realçar pelo contraste. ${ }^{3}$ Esses tumores apresentam localizações preferenciais em regiões não comuns ao lipoma intracraniano como terceiro ventrículo, regiões subfrontal e subtemporal. ${ }^{61,68}$

Quando localizado na cisterna quadrigêmina, apresenta diagnóstico diferencial com cisto aracnóideo, cisto da placa tectal, massas tectais (glioma), abscesso supracerebelar, cisto dermoide e epidermoide, cavernoma ossificado tálamo-mesencefálico, meningioma, ruptura de aneurisma do segmento $\mathrm{P}_{4}$ da artéria cerebral posterior e, mais raramente, com lesões na região da pineal. ${ }^{4,72}$

\section{Tratamento}

O tratamento inicial é conservador, principalmente quando são assintomáticos ou achados incidentais de imagem. . $^{3,49,14,51,64}$ Os LIC, em sua maioria, são pequenos e raramente causam sintomas neurológicos, portanto sua excisão cirúrgica está raramente indicada. ${ }^{72,71}$ Os riscos de uma intervenção cirúrgica superam de longe os benefícios. Isso porque as tentativas de excisão completa do tumor deverão quase certamente lesionar estruturas neurais ou vasculares, tendo em vista a forte adesão do tumor às estruturas circunvizinhas ${ }^{2,5} \mathrm{O}$ desenvolvimento de lipomas pode, ainda, estar associado a anormalidades vasculares, como hipervascularização e angiomas venosos..$^{58,73}$ Além disso, a maioria dos tumores não causa sintomas que põem em risco a vida dos pacientes, o que justifica a indicação de tratamento conservador na maioria dos casos. ${ }^{6,55,65} \mathrm{Em}$ casos de compressão ou contiguidade com defeito craniano e extracraniano, está indicada cirurgia. ${ }^{1,48}$ Vários autores indicam sua exérese parcial ou total em casos de serem sintomáticos ou volumosos e terem localização anatômica acessível. ${ }^{10,59}$ Os LIC localizados profundamente e com aderência no córtex cerebral ou em ramos da artéria cerebral média dificultam sua remoção, tornando impossível sua remoção parcial ou total. ${ }^{14,72}$

Tem sido indicado o uso de shunt em casos de hidrocefalia e da administração de drogas anticonvulsivantes em presença de epilepsia. ${ }^{2,9,14,16} \mathrm{~A}$ epilepsia associada à LIC é geralmente resistente às drogas anticonvulsivantes e não responde bem ao tratamento cirúrgico. ${ }^{31,68,71,74}$ Zettner e Netsky ${ }^{31}$ associaram as crises convulsivas relacionadas à LIC com a infiltração da lesão no parênquima cerebral de maneira progressiva. Segundo Yilmaz et al. ${ }^{14}$ indicação cirúrgica tem sido realizada em casos de sintomas clínicos não responsivos ao tratamento clínico como vertigem e neuralgia trigeminal, porém permanece controversa para diversos autores. 


\section{Considerações finais}

O lipoma intracraniano é uma malformação rara e benigna, resultante de alterações do desenvolvimento, e encontra-se frequentemente associado a disrafismos. Geralmente é assintomático ou um achado incidental de exame de imagem. Com os avanços dos métodos de diagnóstico por imagem, houve aumento da probabilidade de detecção dessas lesões durante a vida, mesmo em pacientes assintomáticos. Por outro lado, seu diagnóstico deve guiar para uma busca por outras anormalidades cerebrais, especialmente na linha média. Seu tratamento é conservador nos casos assintomáticos ou de achado incidental.

\section{Referências}

1. Baeesa SS, Higgins MJ, Ventureyra EC. Dorsal brain stem lipomas: case report. Neurosurgery. 1996;38(5):1031-5.

2. Fandiño J. [Intracranial lipomas]. Rev Neurol. 2001;32(7):64450.

3. Jabot G, Stoquart-Elsankari S, Saliou G, Toussaint $P$, Deramond H, Lehmann P. Intracranial lipomas: clinical appearances on neuroimaging and clinical significance. $J$ Neurol. 2009;256(6):851-5.

4. Ogbole G, Kazaure I, Anas I. Quadrigeminal plate cistern lipoma. BMJ Case Rep. 2009; doi:pii: bcr07.2009.2110. 10.1136/bcr.07.2009.2110.

5. Popa RT, Feier D, Fufezan O, Blaga L. Interhemispheric lipoma associated with agenesis of corpus callosum in an infant: case report. Med Ultrason. 2010;12(3):249-52.

6. Yildiz H, Hakyemez B, Koroglu M, Yesildag A, Baykal B. Intracranial lipomas: importance of localization. Neuroradiology. 2006;48(1):1-7.

7. Zamora AR, Asconape J. Intracranial lipomas: Radiographic and clinical characteristics. Int J Neurol. 2010;12(2):2-6.

8. Cherian A, Baheti NN, Menon R, lyer RS. Hemispheric intracranial lipoma with seizure: look under the carpet. Neurol India. 2011;59(1):128-30.

9. Gómez-Gosálvez FA, Menor-Serrano F, Téllez de MenesesLorenzo M, Aleu Pérez-Gramunt M, Sala-Sánchez AG, Rubio-Soriano A, et al. [Intracranial lipomas in paediatrics: a retrospective study of 20 patients]. Rev Neurol. 2003;37(6):515-21.

10. Hansch A, Mentzel HJ, Boettcher J, Kaiser WA. Intracranial lipoma. A rare congenital malformation. Clin Neuroradiol. 2007;17:52-4.

11. Ichikawa T, Kumazaki T, Mizumura S, Kijima T, Motohashi S, Gocho G. Intracranial lipomas: demonstration by computed tomography and magnetic resonance imaging. J Nippon Med Sch. 2000;67(5):388-91.

12. Venkatesh SK, Phadke RV, Kumar S, Mishra UK. MR appearance of interpeduncular lipoma. Singapore Med J. 2003;44(1):39-41.

13. Wilberger JE Jr, Abla A, Rothfus W. Lipoma of the septum pellucidum: case report. J Comput Tomogr. 1987;11(1):79-82.

14. Yilmaz N, Unal O, Kiymaz N, Yilmaz C, Etlik O. Intracranial lipomas - a clinical study. Clin Neurol Neurosurg. 2006;108(4):363-8.
15. Eghwrudjakpor PO, Kurisaka M, Fukuoka M, Mori K. Intracranial lipomas: current perspectives in their diagnosis and treatment. Br J Neurosurg. 1992;6(2):13944.

16. Maiuri F, Cirillo S, Simonetti L, De Simone MR, Gangemi M. Intracranial lipomas. Diagnostic and therapeutic considerations. J Neurosurg Sci. 1988;32(4):161-7.

17. Puvabanditsin S, Garrow E, Applewhite L, Akpalu D, Quizon MC. Intracranial lipomas in neonate. J Perinatol. 2002;22(5):414-5

18. Fisher RM, Cremin BJ. Lipoma of the corpus callosum: diagnosis by ultrasound and magnetic resonance. Pediatr Radiol. 1988;18(5):409-10.

19. Lin KL, Wang HS, Lui TN. Sonographic diagnosis of a corpus callosum lipoma with extracranial extension in an infant. J Ultrasound Med. 1995;14(7):537-41.

20. Rego I, Safronova MM, Alves D, Pereira JR. Lipomas da linha média. Acta Med Port. 2009;22(2):175.

21. Rokitansky C. Lehrbuch der pathologischen anatomie. Vienna: Braumuller; 1856.

22. Donati F, Vassella F, Kaiser G, Blumberg A. Intracranial lipomas. Neuropediatrics. 1992;23(1):32-8.

23. Kash F, Brown G, Smirniootopoulos JA, Boyer R, Osborn AG. Intracranial lipomas: pathology and imaging spectrum. Int J Neuroradiol. 1996;2:109-16.

24. Imaizumi SO, Pleasure JR, Zubrow AB. Lesion mistaken for hemorrhage in a premature infant: lipoma of corpus callosum. Pediatr Neurol. 1988;4(5):313-6.

25. Truwit CL, Barkovich AJ. Pathogenesis of intracranial lipoma: an MR study in 42 patients. AJR Am J Roentgenol. 1990;155(4):855-64.

26. Vade A, Horowitz SW. Agenesis of corpus callosum and intraventricular lipomas. Pediatr Neurol. 1992;8(4):307-9.

27. Beșkonakli E, Cayli SR, Ergün R, Okten Al. Lipoma of the interpeduncular fossa: demonstration by $\mathrm{CT}$ and MRI. Neurosurg Rev. 1998;21(2-3):210-2.

28. Gastaut H, Regis H, Gastaut JL, Yermenos E, Low MD. Lipomas of the corpus callosum and epilepsy. Neurology. 1980;30(2):132-8.

29. Kieslich M, Ehlers S, Bollinger M, Jacobi G. Midline developmental anomalies with lipomas in the corpus callosum region. J Child Neurol. 2000;15(2):85-9.

30. Sarioğlu AC, Kaynar MY, Hanci M, Uzan M. Sylvian fissure lipomas: case reports and review of the literature. $\mathrm{Br} \mathrm{J}$ Neurosurg. 1999;13(4):386-8.

31. Zettner A, Netsky MG. Lipoma of the corpus callosum. J Neuropathol Exp Neurol. 1960;19:305-19.

32. Spallone A, Pitskhelauri DI. Lipomas of the pineal region. Surg Neurol. 2004;62(1):52-8.

33. Sasaki H, Yoshida K, Wakamoto H, Otani M, Toya S. Lipomas of the frontal lobe. Clin Neurol Neurosurg. 1996;98(1):27-31.

34. Christensen WN, Long DM, Epstein Jl. Cerebellopontine angle lipoma. Hum Pathol. 1986;17(7):739-43.

35. Pensak ML, Glasscock ME 3rd, Gulya AJ, Hays JW, Smith HP, Dickens JR. Cerebellopontine angle lipomas. Arch Otolaryngol Head Neck Surg. 1986;112(1):99-101.

36. Ramos S, Ramos RF, Sad DA, Nogueira HA, Ramos HF, Ramos BF. Lipoma no ângulo ponto-cerebelar: relato de caso e revisão da literatura. Acta ORL. 2005:23(2):13-6.

37. Romero-Blanco M, Monteiro-Santos E. [Cerebellopontine angle lipoma: a case report]. Rev Neurol. 2004;39(3):238-40.

38. Steimlé R, Pageaut G, Jacquet G, Bourghli A, Godard J, Bertaud M. Lipoma in the cerebellopontine angle. Surg Neurol. 1985;24(1):73-6. 
39. Tankéré F, Vitte E, Martin-Duverneuil N, Soudant J. Cerebellopontine angle lipomas: report of four cases and review of the literature. Neurosurgery. 2002;50(3):626-31.

40. Buxi TB, Mathur RK, Doda SS. Computed tomography of lipoma of corpus callosum and choroid plexus lipoma: report of two cases. J Comput Tomogr. 1987;11(1):57-60.

41. Sener RN. Isolated choroid plexus lipomas. Comput Med Imaging Graph. 1995;19(5):423-6.

42. Uchino A, Hasuo K, Matsumoto S, Masuda K. Solitary choroid plexus lipomas: CT and MR appearance. AJNR Am J Neuroradiol. 1993;14(1):116-8.

43. Yock DH Jr. Choroid plexus lipomas associated with lipoma of the corpus callosum. J Comput Assist Tomogr. 1980;4(5):678-82.

44. Schmid AH. A lipoma of the cerebellum. Acta Neuropathol. 1973;26(1):75-80.

45. Cho DY, Wang YC, Li CS, Chang SM. Intracranial lipoma in the medulla oblongata. Surg Neurol. 1991;36(5):384-7.

46. Uchino A, Hasuo K, Matsumoto S, Masuda K. MRI of dorsal mesencephalic lipomas. Clin Imaging. 1993;17(1):12-6.

47. Chen CF, Lee YC, Lui CC, Lee RJ. Posterior pericallosal lipoma extending through the interhemispheric fissure into the scalp via the anterior fontanelle. Neuroradiology. 2004;46(8):692-5.

48. Kudoh H, Sakamoto K, Kobayashi N. Lipomas in the corpus callosum and the forehead, associated with a frontal bone defect. Surg Neurol. 1984;22(5):503-8.

49. Mitilian D, Haddad D, Lenoir M, Boudjemaa S, Vazquez MP, Picard A. Interhemispheric lipoma associated with frontal subcutaneous lipoma. J Plast Reconstr Aesthet Surg. 2009;62(11):e427-9.

50. Sari A, Dinç H, Gümele HR. Interhemispheric lipoma associated with subcutaneous lipoma. Eur Radiol. 1998;8(4):628-30.

51. Yilmazlar S, Kocaeli H, Aksoy K. Quadrigeminal cistern lipoma. J Clin Neurosci. 2005;12(5):596-9.

52. Budka H. Intracranial lipomatous hamartomas (intracranial "lipomas"). A study of 13 cases including combinations with medulloblastoma, colloid and epidermoid cysts, angiomatosis and other malformations. Acta Neuropathol. 1974;28(3):205-22.

53. Hori A. Lipoma of the quadrigeminal region with evidence of congenital origin. Arch Pathol Lab Med. 1986;110(9):850-1.

54. Martínez-Lapiscina EH, García MP, Alegría MB. [Epileptic seizure and lipoma of corpus callosum: cause or incidental finding]. Neurologia. 2010;25(5):331-2.

55. Given CA, Fields TM, Pittman T. Interhemispheric lipoma connected to subcutaneous lipoma via lipomatous stalk. Pediatr Radiol. 2005;35(11):1110-2.

56. Fitoz S, Atasoy C, Erden I, Akyar S. Intracranial lipoma with extracranial extension through foramen ovale in a patient with encephalocraniocutaneous lipomatosis syndrome. Neuroradiology. 2002;44(2):175-8.

57. Moog U, Jones MC, Viskochil DH, Verloes A, Van Allen MI, Dobyns WB. Brain anomalies in encephalocraniocutaneous lipomatosis. Am J Med Genet A. 2007;143(24):2963-72.

58. Ahmetoglu A, Aynaci FM, Sari A. Sylvian fissure lipoma associated with cortical dysplasia and abnormal vascularity. Eur J Radiol. 2003;46(2):43-6.
59. Kim JY, Lee TJ, Chi JG. Congenital quadrigeminal lipoma with osteocartilagenous element. A case report. J Korean Med Sci. 1996;11(6):537-9.

60. Fujii T, Takao T, Ito M, Konishi Y, Okuno T, Suzuki J. Lipoma of the corpus callosum: a case report with a review. Comput Radiol. 1982;6(5):301-4.

61. Halmagyi GM, Evans WA. Lipoma of the quadrigeminal plate causing progressive obstructive hydrocephalus. Case report. J Neurosurg. 1978;49(3):453-6.

62. Ono J, Ikeda T, Imai K, Mano T, Matsuoka T, Nagai T, et al. Intracranial lipoma of the quadrigeminal region associated with complex partial seizures. Pediatr Radiol. 1998;28(9):729-31.

63. Yilmazlar S, Kocaeli H, Aksoy K. Quadrigeminal cistern lipoma. J Clin Neurosci. 2005;12(5):596-9.

64. Loddenkemper T, Morris HH 3rd, Diehl B, Lachhwani DK. Intracranial lipomas and epilepsy. J Neurol. 2006;253(5):590-3.

65. Hädecke J, Buchfelder M, Triebel HJ, Schneyer U. Multiple intracranial lipoma: a case report. Neurosurg Rev. 1997;20(4):282-7.

66. Zimmermann M, Kellermann S, Gerlach R, Seifert V. Cerebellopontine angle lipoma: case report and review of the literature. Acta Neurochir (Wien). 1999;141(12):1347-51.

67. Friedman RB, Segal R, Latchaw RE. Computerized tomographic and magnetic resonance imaging of intracrania lipoma. Case report. J Neurosurg. 1986;65(3):407-10.

68. Kazner E, Stochdorph O, Wende S, Grumme T. Intracranial lipoma. Diagnostic and therapeutic considerations. J Neurosurg. 1980;52(2):234-45.

69. Shinozaki N, Sekiya T, Suzuki S, Iwabuchi T, Suzuki M. [Five cases of intracranial lipoma; CT and magnetic resonance images]. No Shinkei Geka. 1992;20(3):289-93.

70. Olm M, Blesa R, Ribera G, Cardenal C. Lipoma of the corpus callosum: CT and MR for diagnosis. AJR Am J Roentgenol. 1988;151(3):613-4.

71. Piovesan EJ, Tatsui CE, Kowacs PA, Prazeres RF, Lange MC, Antoniuk SA, et al. [Lipoma of the corpus callosum associated with the hypertrophy of the corpus callosum: case report]. Arq Neuropsiquiatr. 2000;58(3B):947-51.

72. Nikaido $\mathrm{Y}$, Imanishi M, Monobe T. Lipoma in the quadrigeminal cistern - case report. Neurol Med Chir (Tokyo). 1995;35(3):175-8.

73. Kakita A, Inenaga C, Kameyama S, Masuda H, Ueno T, Honma J, et al. Cerebral lipoma and the underlying cortex of the temporal lobe: pathological features associated with the malformation. Acta Neuropathol. 2005;109(3):339-45.

74. Wallace D. Lipoma of the corpus callosum. J Neurol Neurosurg Psychiatry. 1976;39(12):1179-85.

Endereço para correspondência

Carlos Umberto Pereira

Av. Augusto Maynard, 245/404, Bairro São José

49015-380 - Aracaju, SE, Brasil

E-mail: umberto@infonet.com.br 